\title{
Ruptured brachial artery mycotic aneurysm in kidney transplant recipient: a case report
}

\author{
Hojong Park ${ }^{1}$, Sang Jun Park ${ }^{1}$, Hong Rae Cho ${ }^{1}$, Kyung Sun Park², Jongha Park², Kyung Don Yoo ${ }^{2}$, Jong Soo Lee ${ }^{2}$ \\ ${ }^{1}$ Department of Surgery, Ulsan University Hospital, Ulsan, Korea \\ ${ }^{2}$ Department of Internal Medicine-Nephrology, Ulsan University Hospital, Ulsan, Korea
}

Background: Brachial artery mycotic aneurysm is a rare condition. It can be a complication of hematogenous spread of bacterial infection. We report a case of ruptured brachial artery mycotic aneurysm in kidney transplant recipient.

Case report: A 62-year-old male patient who had kidney transplantation for end-stage renal disease was admitted with left arm pain and swelling. He had deceased donor kidney transplantation 5 years ago and he had left brachiocephalic fistula ligation 3 years previously. One month ago, the patient was hospitalized and treated for urinary tract infection. Urine culture revealed ESBL (-) Escherichia coli. His condition improved and he was prescribed antibiotics from the outpatient department. One week before admission, he was vaccinated on his left shoulder. The cause of his arm pain and swelling was likely cellulitis or deep vein thrombosis; we performed imaging studies. US Doppler and computed tomography angiography showed a thrombosed occluded brachial artery aneurysm measuring $25 \mathrm{~mm}$ in diameter and $18 \mathrm{~cm}$ in length throughout the brachial artery. The radio-ulnar bifurcation site was reconstituted by two early branching collateral arteries above the brachial artery aneurysm. We performed aneurysm resection and two early branching collateral arteries were preserved, one of which required reanastomosis. Brachial artery aneurysm revealed contained rupture and severe adhesion to adjacent tissues. Brachial artery microbiological examination revealed E.coli.

Conclusions: Brachial artery mycotic aneurysm can be potentially limb or life threatening. It is important to acknowledge urinary tract infection as a cause. The best therapeutic management is surgical repair after a prompt diagnosis.

Corresponding author: Sang Jun Park

E-mail: sjpark@uuh.ulsan.kr

\footnotetext{
(c) The Korean Society for Transplantation

This is an Open Access article distributed under the terms of the Creative Commons Attribution Non-Commercial License (http://creativecommons.org/licenses/by-nc/4.0/) which permits unrestricted non-commercial use, distribution, and reproduction in any medium, provided the original work is properly cited.
} 